VOL. $62(2000)$ [119-134]

\title{
THE AVERAGE DISTANCE PROPERTY OF CLASSICAL BANACH SPACES
}

\author{
AICKE HINRICHS
}

A Banach space $X$ has the average distance property (ADP) if there exists a unique real number $r$ such that for each positive integer $n$ and all $x_{1}, \ldots, x_{n}$ in the unit sphere of $X$ there is some $x$ in the unit sphere of $X$ such that

$$
\frac{1}{n} \sum_{k=1}^{n}\left\|x_{k}-x\right\|=r .
$$

It is known that $l_{2}$ and $l_{\infty}$ have the ADP, whereas $l_{p}$ fails to have the ADP if $1 \leqslant p<2$. We show that $l_{p}$ also fails to have the ADP for $3 \leqslant p \leqslant \infty$. Our method seems to be able to decide also the case $2<p<3$, but the computational difficulties increase as $p$ comes closer to 2 .

\section{INTRODUCTION}

A rendezvous number of a metric space $(M, d)$ is a real number $r$ with the property that for each positive integer $n$ and $x_{1}, \ldots, x_{n} \in M$ there exists $x \in M$ such that

$$
\frac{1}{n} \sum_{k=1}^{n} d\left(x_{k}, x\right)=r \text {. }
$$

A remarkable theorem of Gross [2] states that any compact connected metric space has a unique rendezvous number. A nice survey of this subject is given in [1]. We say that a (real or complex) Banach space $X$ has the average distance property (ADP) if its unit sphere $S(X)$ has a unique rendezvous number. In this case, we say that this is also the rendezvous number of $X$. The Gross theorem implies that a finite dimensional (dimension $\geqslant 2$ if the scalars are real) Banach space has the ADP. Observe that 1 is the unique rendezvous number of the unit ball of any Banach space, see [6].

Let $\mathbb{K}$ stand as a synonym for the scalar field $\mathbb{R}$ or $\mathbb{C}$. For $1 \leqslant p \leqslant \infty$ and $d \in \mathbb{N}$, let $l_{p}^{d}(\mathbb{K})$ be $\mathbb{K}^{d}$ equipped with the usual $p$-norm. If a statement holds for both choices of the scalar field we simply write $l_{p}^{d}$. Since we want to deal with connected unit spheres

Received 18th October, 1999

Supported by DFG grants Pi 322/1-2 and Hi 584/1-1.

Copyright Clearance Centre, Inc. Serial-fee code: 0004-9727/00 \$A2.00+0.00. 
only we always assume $d \geqslant 2$ in the real case. Further, let $l_{p}(\mathbb{K})$ be the space of all scalar sequences with finite $p$-norm and let $L_{p}=L_{p}[0,1]$ be the space of all (equivalence classes of) scalar functions on the interval $[0,1]$ with finite $p$-norm.

It is shown in $[4,6,5]$ that

- $l_{2}$ has the ADP with rendezvous number $\sqrt{2}$.

- $l_{\infty}(\mathbb{R})$ and $l_{\infty}^{d}(\mathbb{R})$ have the ADP with rendezvous number $3 / 2$.

- $l_{p}$ and $L_{p}$ do not have the ADP if $1 \leqslant p<2$.

Most of this article is devoted to the proof of the following theorem.

THEOREM 1.1. For $3 \leqslant p<\infty, l_{p}$ and $L_{p}$ do not have the ADP.

In section 7 , we show that complex $l_{\infty}$ has the ADP, more precisely we have

THEOREM 1.2. $l_{\infty}(\mathbb{C})$ and $l_{\infty}^{d}(\mathbb{C})$ have the $A D P$ with rendezvous number $1 / 3+$ $2 \sqrt{3} / \pi$.

Both theorems answer questions raised in [5]. We were not able to decide whether $l_{p}$ and $L_{p}$ enjoy the ADP for $2<p<3$, but our methods strongly suggest that they do not. Our method traces the question back to the proof of some elementary inequalities. Nevertheless, the computational difficulties, for example, the number of variables involved, increases as $p$ comes closer to 2. We comment on the situation in more detail in Section 6 .

\section{THE AVERAGE DISTANCE PROPERTY OF $l_{p}$ FOR $p>2$}

For $l_{p}, 1 \leqslant p<\infty$, the next fact was observed in [5]. To keep this paper selfcontained, we give a proof covering both the sequence and the function space case.

Proposition 2.1. For $1 \leqslant p<\infty$, the only possible rendezvous number of $l_{p}$ or $L_{p}$ is $\sqrt[p]{2}$.

Proof: Let $X=l_{p}$ or $X=L_{p}$. For $N \in \mathbb{N}$ to be chosen later, let $x_{1}, \ldots, x_{N} \in S(X)$ be disjointly supported vectors and choose a further vector $x \in S(X)$. We show that, for $N$ large enough, $\sum_{k=1}^{N}\left\|x_{k}-x\right\| / N$ is arbitrarily close to $\sqrt[p]{2}$ which proves the proposition.

Let $\Delta_{k}$ be the support of $x_{k}$ and denote $\sigma_{k}=\left\|\left.x\right|_{\Delta_{k}}\right\|$. Since

$$
\left\|x_{k}-x\right\|^{p}=1-\left\|\left.x\right|_{\Delta_{k}}\right\|^{p}+\left\|x-\left.x\right|_{\Delta_{k}}\right\|^{p},
$$

we obtain from

$$
1-\sigma_{k} \leqslant\left\|x-\left.x\right|_{\Delta_{k}}\right\| \leqslant 1+\sigma_{k}
$$

that

$$
\frac{1}{N} \sum_{k=1}^{N} \phi_{-}\left(\sigma_{k}\right) \leqslant \frac{1}{N} \sum_{k=1}^{N}\left\|x_{k}-x\right\| \leqslant \frac{1}{N} \sum_{k=1}^{N} \phi_{+}\left(\sigma_{k}\right)
$$


where

$$
\phi_{ \pm}(\sigma)=\sqrt[p]{1-\sigma^{p}+(1 \pm \sigma)^{p}}
$$

These functions are continuous, so, given $\varepsilon>0$, we can choose $\delta>0$ such that $\mid \phi_{ \pm}(\sigma)-$ $\sqrt[p]{2} \mid<\varepsilon / 2$ provided that $0 \leqslant \sigma \leqslant \delta$. Letting $A=\left\{k=1, \ldots, N: \sigma_{k}>\delta\right\}$, we obtain from (1) that

$$
\frac{N-|A|}{N}\left(\sqrt[8]{2}-\frac{\varepsilon}{2}\right) \leqslant \frac{1}{N} \sum_{k=1}^{N}\left\|x_{k}-x\right\| \leqslant \frac{N-|A|}{N}\left(\sqrt[p]{2}+\frac{\varepsilon}{2}\right)+\frac{2|A|}{N} .
$$

By disjointness of $\Delta_{k}$ and $\|x\|=1$, we conclude that $\sum_{k=1}^{N} \sigma_{k}^{p} \leqslant 1$ which in turn implies $|A| \leqslant 1 / \delta^{p}$. Substituting this cardinality estimate into (2) yields

$$
\sqrt[p]{2}-\frac{\varepsilon}{2}-\frac{\sqrt[p]{2}-\varepsilon / 2}{N \delta^{p}} \leqslant \frac{1}{N} \sum_{k=1}^{N}\left\|x_{k}-x\right\| \leqslant \sqrt[p]{2}+\frac{\varepsilon}{2}+\frac{2}{N \delta^{p}} .
$$

It is left to choose $N$ large enough to ensure that

$$
\sqrt[p]{2}-\varepsilon \leqslant \frac{1}{N} \sum_{k=1}^{N}\left\|x_{k}-x\right\| \leqslant \sqrt[8]{2}+\varepsilon
$$

The next proposition is a consequence of the well-known Borsuk-Ulam theorem. For $d \in \mathbb{N}$, let $\mathbb{S}^{d}$ be the Euclidean unit sphere in $\mathbb{R}^{d+1}$.

Proposition 2.2. Let $X$ be a Banach space, $n \in \mathbb{N}$ and $x_{1}, \ldots, x_{n} \in S(X)$. For any subspace $E$ of $X$ with dimension at least $n+1$ for real scalars and $n / 2+1$ for complex scalars, respectively, there exists $x \in S(E)$ such that $\left\|x_{k}-x\right\|=\left\|x_{k}+x\right\|$ for $k=1, \ldots, n$.

Proof: Define the function $\phi: S(E) \longrightarrow \mathbb{R}^{n}$ by $\phi(x)=\left(\left\|x_{k}-x\right\|\right)$. Let $\psi: \mathbb{S}^{d} \longrightarrow$ $S(E)$ be a homeomorphism such that $\psi(-t)=-\psi(t)$ for $t \in \mathbb{S}^{d}$, where $\mathbf{S}^{d}$ is the Euclidian unit sphere in $\mathbb{R}^{d+1}$. Then $\phi \circ \psi$ is a continuous map from $\mathbb{S}^{d}$ into $\mathbb{R}^{n}$. Since $d \geqslant n$, the Borsuk-Ulam theorem tells us that there is $t \in \mathbb{S}^{d}$ with $\phi \circ \psi(-t)=\phi \circ \psi(t)$. Letting $x=\psi(t)$ yields $\phi(-x)=\phi(x)$ which is the claim of the proposition.

The preceding proposition implies that it is always possible to arrange for an average distance at least $\sqrt[p]{2}$. More precisely, we have the following corollary.

Corollary 2.3. Let $2 \leqslant p<\infty, n \in \mathbb{N}$ and $X=l_{p}$ or $X=L_{p}$. If the scalars are real let $m=n+1$, if the scalars are complex let $m=n / 2+1$. For $x_{1}, \ldots, x_{n} \in X$ and any subspace $E$ of $X$ of dimension at least $m$, there exists $x \in S(E)$ such that

$$
\left\|x_{k}-x\right\|=\left\|x_{k}+x\right\| \geqslant \sqrt[p]{2} \text { for } k=1, \ldots, n .
$$


Proof: Proposition 2.2 gives us $x \in S(E)$ such that $\left\|x_{k}-x\right\|=\left\|x_{k}+x\right\|$ for $k=1, \ldots, n$. So it is enough to verify that $\left\|y_{1}+y_{2}\right\|^{p}+\left\|y_{1}-y_{2}\right\|^{p} \geqslant 2\left(\left\|y_{1}\right\|^{p}+\left\|y_{2}\right\|^{p}\right)$ for $y_{1}, y_{2} \in X$. Since we are in $l_{p}$ or $L_{p}$, this follows easily from Clarkson's inequality $|u+v|^{p}+|u-v|^{p} \geqslant 2\left(|u|^{p}+|v|^{p}\right)$ which is valid for all $u, v \in \mathbb{C}$. Indeed, by Hölder's inequality

$$
|u+v|^{p}+|u-v|^{p} \geqslant 2^{1-p / 2}\left(|u+v|^{2}+|u-v|^{2}\right)^{p / 2}=2\left(|u|^{2}+|v|^{2}\right)^{p / 2} \geqslant 2\left(|u|^{p}+|v|^{p}\right) .
$$

It is the purpose of the next sections to find, for $p \geqslant 3$ and $X=l_{p}$ or $X=L_{p}$, vectors $x_{1}, \ldots, x_{n} \in S(X)$ such that

$$
\frac{1}{N} \sum_{k=1}^{N}\left\|x_{k}-x\right\|>\sqrt[p]{2} \text { for all } x \in S(X) .
$$

\section{Sharpening Clarkson's inequality}

For the next two sections, let $p>2$ be fixed and let $X=l_{p}$ or $X=L_{p}$. We want to give good lower estimates for $\|x+y\|$ in terms of $\|x\|,\|y\|$ and $\|x-y\|$, where $x, y \in X$. These inequalities are used in the next section to play the question whether $X$ has the ADP down to the question whether a certain inequality for real numbers holds. Nevertheless, they seem to be of some independent interest since they sharpen Clarkson's inequality considerably.

Clarkson's inequality, as already used in the proof of Corollary 2.3, says that

$$
\|x+y\|^{p} \geqslant 2\|x\|^{p}+2\|y\|^{p}-\|x-y\|^{p} \text { whenever } x, y \in X .
$$

Unfortunately, this inequality is not strong enough for our purposes. So we shall look for a function $\phi$ such that

$$
\|x+y\|^{p} \geqslant \phi\left(\|x\|^{p}+\|y\|^{p},\|x-y\|^{p}\right) \text { whenever } x, y \in X
$$

which is better than (3). Observe that (3) corresponds to the affine function $\phi(s, t)=$ $2 s-t$. It is natural to assume that $\phi$ is homogeneous. The next proposition makes it easy to identify such functions. Let us agree that 0 multiplied by something undefined is again 0 .

Proposition 3.1. Let $f:\left[0,2^{p-1}\right] \rightarrow\left[0,2^{p-1}\right]$ be a convex continuous function such that

$$
f\left(|\xi-\eta|^{p}\right) \leqslant|\xi+\eta|^{p} \text { for all } \xi, \eta \in \mathbb{R} \text { with }|\xi|^{p}+|\eta|^{p}=1
$$

Define $\phi$ by $\phi(s, t)=s f(t / s)$ for $0 \leqslant t \leqslant 2^{p-1} s$. Then

$$
\|x+y\|^{p} \geqslant \phi\left(\|x\|^{p}+\|y\|^{p},\|x-y\|^{p}\right) \text { whenever } x, y \in X .
$$


Proof: Let us first check that (5) for all reals already ensures that the same is true for all complex numbers $\xi$ and $\eta$. We may assume that $|\xi| \geqslant|\eta|$. We can choose $\alpha, \beta \in \mathbf{R}$ such that $|\alpha|^{p}+|\beta|^{p}=1, \alpha \geqslant|\beta|$ and $|\xi-\eta|=\alpha-\beta$. Since we have (5) for $\alpha$ and $\beta$, it is enough to show that $|\xi+\eta| \geqslant \alpha+\beta$. To this end, choose $u \in[-1,1]$ and $v \in[0,1]$ such that

$$
\alpha=\frac{1}{\left(1+|u|^{p}\right)^{1 / p}}, \quad \beta=\frac{u}{\left(1+|u|^{p}\right)^{1 / p}}, \quad|\xi|=\frac{1}{\left(1+v^{p}\right)^{1 / p}} \text { and }|\eta|=\frac{v}{\left(1+v^{p}\right)^{1 / p}}
$$

Since the function $(1-u) /\left(1+|u|^{p}\right)^{1 / p}$ is decreasing in $u \in[-1,1]$, we infer from $|\xi|-|\eta| \leqslant$ $\alpha-\beta \leqslant|\xi|+|\eta|$ that $|u| \leqslant v$. Since the function $\left(1+|u|^{2}\right) /\left(1+|u|^{p}\right)^{2 / p}$ is increasing in $|u| \in[0,1]$, this implies $\alpha^{2}+\beta^{2} \leqslant|\xi|^{2}+|\eta|^{2}$. But then we indeed have

$$
\begin{aligned}
|\xi+\eta|^{2} & =2|\xi|^{2}+2|\eta|^{2}-|\xi-\eta|^{2}=2|\xi|^{2}+2|\eta|^{2}-(\alpha-\beta)^{2} \\
& =(\alpha+\beta)^{2}+2|\xi|^{2}+2|\eta|^{2}-2 \alpha^{2}-2 \beta^{2} \geqslant(\alpha+\beta)^{2} .
\end{aligned}
$$

Now we are in a position to tackle the final statement of the proposition. Since $\varphi$ is continuous, it is enough to prove (6) for $x, y$ with finite support if $X=l_{p}$ and $x, y$ finitely valued if $X=L_{p}$, respectively. So we alternatively may assume that $x=\left(\xi_{k}\right), y=\left(\eta_{k}\right) \in$ $l_{p}^{n}$ for some $n$. Since $\varphi$ is homogeneous, we can also assume that

$$
\|x\|^{p}+\|y\|^{p}=\sum_{k=1}^{n}|\xi|^{p}+|\eta|^{p}=1 .
$$

Then we conclude from (5) for complex numbers and from the convexity of $f$ that

$$
\begin{aligned}
\|x+y\|^{p} & =\sum_{k=1}^{n}\left|\xi_{k}+\eta_{k}\right|^{p} \geqslant \sum_{k=1}^{n}\left(\left|\xi_{k}\right|^{p}+\left|\eta_{k}\right|^{p}\right) f\left(\frac{\left|\xi_{k}-\eta_{k}\right|^{p}}{\left|\xi_{k}\right|^{p}+\left|\eta_{k}\right|^{p}}\right) \\
& \geqslant f\left(\sum_{k=1}^{n}\left|\xi_{k}-\eta_{k}\right|^{p}\right)=f\left(\|x-y\|^{p}\right)=\varphi\left(\|x\|^{p}+\|y\|^{p},\|x-y\|^{p}\right) .
\end{aligned}
$$

Let us now define a function $f$ which serves well for our purposes. Actually, it is the best function which satisfies the conditions imposed in the previous proposition. For $0 \leqslant \sigma \leqslant 2^{p-1}$, there exists a unique $u \in[-1,1]$ such that $\sigma=(1-u)^{p} /\left(1+|u|^{p}\right)$. Then we let $f(\sigma)=(1+u)^{p} /\left(1+|u|^{p}\right)$. Let us first check that $f$ indeed satisfies (5). If $\xi, \eta \in \mathbb{R}$ are such that $|\xi|^{p}+|\eta|^{p}=1$ and $|\xi-\eta|^{p}=\sigma$, and we additionally assume without loss of generality that $\xi>0$ and $\xi^{p} \geqslant 1 / 2$, it is easily seen that

$$
\xi=\frac{1}{\left(1+|u|^{p}\right)^{1 / p}} \text { and } \eta=\frac{u}{\left(1+|u|^{p}\right)^{1 / p}}
$$

where $u$ is determined by $\sigma$ as above. But then

$$
|\xi+\eta|^{p}=(1+u)^{p} /\left(1+|u|^{p}\right)=f\left(|\xi-\eta|^{p}\right),
$$


so we even have equality in (5). Continuity of $f$ is clear from the definition. Let us finally verify that $f$ is also convex, so that the $\phi$ obtained from this $f$ indeed has property (6). We use the parameterisation of the curve $y=f(x)$ by $u \in[-1,1]$ from the definition

$$
x(u)=\frac{(1-u)^{p}}{1+|u|^{p}} \text { and } y(u)=\frac{(1+u)^{p}}{1+|u|^{p}} .
$$

Since the curve is symmetric about the line $y=x$, it is enough to prove that the curve is convex in the parameter interval $u \in[0,1]$ and that the left derivative at the point $(1,1)$ corresponding to the parameter value $u=0$ equals -1 . It is an elementary calculation to show that, for $u \in[0,1]$,

$$
\frac{d f}{d x}(x(u))=\frac{y^{\prime}(u)}{x^{\prime}(u)}=-\frac{(1-u)^{p-1}}{1-u^{p-1}} \frac{1+u^{p-1}}{(1+u)^{p-1}} .
$$

So we indeed have $f^{\prime}(1)=-1$. Moreover, it is one more exercise in elementary calculus to show that the functions

$$
\psi_{1}(u)=\frac{(1+u)^{\alpha}}{1+u^{\alpha}} \quad \text { and } \quad \psi_{2}(u)=\frac{(1-u)^{\alpha}}{1-u^{\alpha}}
$$

are monotonically increasing and decreasing, respectively, in $[0,1]$ for $\alpha>1$. Hence $\frac{d f}{d x}(x(u))$ is monotonically increasing in $u \in[0,1]$. Since $x$ decreases as $u$ increases, we find that $f$ is indeed convex.

Corollary 3.2. Let $f:\left[0,2^{p-1}\right] \rightarrow\left[0,2^{p-1}\right]$ be the function defined above. Define $\phi$ by $\phi(s, t)=s f(t / s)$ for $0 \leqslant t \leqslant 2^{p-1} s$. Then

$$
\|x+y\|^{p} \geqslant \phi\left(\|x\|^{p}+\|y\|^{p},\|x-y\|^{p}\right) \text { whenever } x, y \in X .
$$

\section{Estimating MEAN Distances}

For $n=2,3, \ldots$, let $\mathbb{D}_{n}=\left\{\varepsilon=\left(\varepsilon_{1}, \ldots, \varepsilon_{n}\right): \varepsilon_{i}= \pm 1\right\}$ be the set of all vectors of $n$ signs. Let $x \in X$ satisfy $n\|x\|^{p}=1$. Now we define, for each $\varepsilon \in \mathbb{D}_{n}$, a vector $x_{\varepsilon} \in S(X)$. If $X=l_{p}$ and $x=\left(\xi_{j}\right)$, we let $x_{\varepsilon}(n(j-1)+i)=\varepsilon_{i} \xi_{j}$ for $i=1, \ldots, n$ and $j=1,2, \ldots$, that is

$$
x_{\varepsilon}=\left(\varepsilon_{1} \xi_{1}, \ldots, \varepsilon_{n} \xi_{1}, \varepsilon_{1} \xi_{2}, \ldots, \varepsilon_{n} \xi_{2}, \ldots\right)
$$

If $X=L_{p}$, we let

$$
x_{\varepsilon}(t)=n^{1 / p} \varepsilon_{i} x(n t-i+1) \text { for } t \in[(i-1) / n, i / n) \text { and } i=1, \ldots, n \text {. }
$$

Moreover, given $y \in X$, we define vectors $y_{1}, \ldots, y_{n} \in X$ by the following rule. If $X=l_{p}$ and $y=\left(\eta_{j}\right)$, we let $y_{i}(j)=\eta_{n(j-1)+i}$. If $X=L_{p}$, we let $y_{i}(t)=n^{-1 / p} y((i-1+t) / n)$. In either case, we obtain that

$$
\|y\|^{p}=\sum_{i=1}^{n}\left\|y_{i}\right\|^{p} \quad \text { and } \quad\left\|x_{\varepsilon}-y\right\|^{p}=\sum_{i=1}^{n}\left\|x-\varepsilon_{i} y_{i}\right\|^{p} .
$$


By symmetry, we conclude that

$$
\frac{1}{2^{n}} \sum_{\varepsilon \in \mathbf{D}_{n}}\left\|x_{\varepsilon}-y\right\|=\frac{1}{2^{n}} \sum_{\varepsilon \in \mathbb{D}_{n}}\left(\sum_{i=1}^{n}\left\|x+\varepsilon_{i} y_{i}\right\|^{p}\right)^{1 / p} .
$$

We are going to show that, for $p \geqslant 3$ there exists $x \in X$ with $n\|x\|^{p}=1$ such that this expression is strictly bigger than $\sqrt[p]{2}$ for all $y \in S(X)$. In fact, for $p \geqslant 3$ even $n=2$ works. This will prove Theorem 1.1.

To this end, we use the functions $f$ and $\phi$ as defined in the preceding section. Let us write $A \leqslant n$ to abbreviate $A \subset\{1, \ldots, n\}$. Then we can estimate the average in (8) using Corollary (3.2) together with the definition of $\phi$ as follows.

$$
\begin{aligned}
& \frac{1}{2^{n}} \sum_{\varepsilon \in \mathbb{D}_{n}}\left(\sum_{i=1}^{n}\left\|x+\varepsilon_{i} y_{i}\right\|^{p}\right)^{1 / p}=\frac{1}{2^{n}} \sum_{A \leqslant n}\left(\sum_{i \in A}\left\|x+y_{i}\right\|^{p}+\sum_{i \notin A}\left\|x-y_{i}\right\|^{p}\right)^{1 / p} \\
& \geqslant \frac{1}{2^{n}} \sum_{A \leqslant n}\left(\sum_{i \in A} \phi\left(\|x\|^{p}+\left\|y_{i}\right\|^{p},\left\|x-y_{i}\right\|^{p}\right)+\sum_{i \notin A}\left\|x-y_{i}\right\|^{p}\right)^{1 / p} \\
& =\frac{1}{2^{n}} \sum_{A \leqslant n}\left(\sum_{i \in A}\left(\|x\|^{p}+\left\|y_{i}\right\|^{p}\right) f\left(\frac{\left\|x-y_{i}\right\|^{p}}{\|x\|^{p}+\left\|y_{i}\right\|^{p}}\right)+\sum_{i \notin A}\left\|x-y_{i}\right\|^{p}\right)^{1 / p} .
\end{aligned}
$$

Let us set

$$
\sigma_{i}=\frac{\left\|x-y_{i}\right\|^{p}}{\|x\|^{p}+\left\|y_{i}\right\|^{p}} \quad \text { and } \quad \alpha_{i}=\frac{\|x\|^{p}+\left\|y_{i}\right\|^{p}}{2} \text { for } i=1, \ldots, n .
$$

Then we can further write

$$
\frac{1}{2^{n}} \sum_{\varepsilon \in \mathbb{D}_{n}}\left(\sum_{i=1}^{n}\left\|x+\varepsilon_{i} y_{i}\right\|^{p}\right)^{1 / p} \geqslant \frac{\sqrt[p]{2}}{2^{n}} \sum_{A \leqslant n}\left(\sum_{i \in A} \alpha_{i} f\left(\sigma_{i}\right)+\sum_{i \notin A} \alpha_{i} \sigma_{i}\right)^{1 / p} .
$$

Taking into account the already observed formula $f(f(\sigma))=\sigma$ and the symmetry of the last equation, we may assume that $\sigma_{i} \in[0,1]$ for $i=1, \ldots, n$. Let $u_{i} \in[0,1]$ be the corresponding parameter value in the definition of $f$, so that we have $\sigma_{i}=\left(1-u_{i}\right)^{p} /\left(1+u_{i}^{p}\right)$ and $f\left(\sigma_{i}\right)=\left(1+u_{i}\right)^{p} /\left(1+u_{i}^{p}\right)$. Then we arrive at the inequality

$$
\frac{1}{2^{n}} \sum_{\varepsilon \in D_{n}}\left(\sum_{i=1}^{n}\left\|x+\varepsilon_{i} y_{i}\right\|^{p}\right)^{1 / p} \geqslant \frac{\sqrt[p]{2}}{2^{n}} \sum_{\varepsilon \in \mathbf{D}_{n}}\left(\sum_{i=1}^{n} \alpha_{i} \frac{\left(1+\varepsilon_{i} u_{i}\right)^{p}}{1+u_{i}^{p}}\right)^{1 / p} .
$$

Let us now assume that $y \in S(X)$. Then we conclude from (7) and $n\|x\|^{p}=1$ that

$$
\sum_{i=1}^{n} \alpha_{i}=1 \text { and } \frac{1}{2 n} \leqslant \alpha_{i} \leqslant \frac{1}{2}+\frac{1}{2 n} .
$$


So we finally obtain from (8) that

$$
\frac{1}{2^{n}} \sum_{\varepsilon \in \mathbf{D}_{n}}\left\|x_{\varepsilon}-y\right\| \geqslant \sqrt[p]{2} \min \phi_{p}(\alpha, u)
$$

where

$$
\phi_{p}(\alpha, u)=\frac{1}{2^{n}} \sum_{\varepsilon \in \mathbb{D}_{n}}\left(\sum_{i=1}^{n} \alpha_{i} \frac{\left(1+\varepsilon_{i} u_{i}\right)^{p}}{1+u_{i}^{p}}\right)^{1 / p}
$$

and the minimum is taken over all $u=\left(u_{1}, \ldots, u_{n}\right) \in[0,1]^{n}$ and $\alpha=\left(\alpha_{1}, \ldots, \alpha_{n}\right)$ subject to the condition (9). We have now traveled most of the way towards the next theorem.

Theorem 4.1. Let $p>2, n \in \mathbb{N}$ and $X=L_{p}$ or $X=l_{p}$. Let $\phi_{p}$ be defined as in (11). Assume that $\phi_{p}(\alpha, u) \geqslant 1$ for all $u \in[0,1]^{n}$ and $\alpha=\left(\alpha_{1}, \ldots, \alpha_{n}\right)$ satisfying (9). Moreover, assume that $\phi_{p}(\alpha, u)=1$ if and only if $u_{1}=\ldots=u_{n}=0$. Then

$$
\frac{1}{2^{n}} \sum_{\varepsilon \in \mathbb{D}_{n}}\left\|x_{\varepsilon}-y\right\| \geqslant \sqrt[p]{2}
$$

for all $x \in X$ with $n\|x\|^{p}=1$ and $y \in S(X)$, where the $x_{\varepsilon}$ are defined as in the beginning of this section. Equality can occur only if the support of $x$ is (essentially) disjoint from the support of all the $y_{i}$ obtained from $y$ as above. In this case $X$ does not have the ADP.

PROOF: It follows from the preceding considerations that the equality case $u_{1}=\ldots=u_{n}$ corresponds to the case when

$$
\left\|x+y_{i}\right\|^{p}=\left\|x-y_{i}\right\|^{p}=\|x\|^{p}+\left\|y_{i}\right\|^{p} \quad \text { for } \quad i=1, \ldots, n .
$$

But this can only happen if the support of $x$ is (essentially) disjoint from the support of each of the $y_{i}$. By choosing an $x$ with full support and concluding from $\|y\|=1$ that at least one of the $y_{i}$ must have (essentially) non-vanishing support, we see that for each $y \in S(X)$ there is strict inequality. But this means that $\sqrt[p]{2}$ can not be a rendezvous number of $X$, so that $X$ can not have the ADP.

\section{VARYING $p$}

That the assumption in Proposition 4.1 can not be satisfied for all $p>2$ with a fixed $n$ can be easily seen by evaluating $\phi_{p}$ for $\alpha_{1}=\ldots=\alpha_{n}=1 / n$ and $u_{1}=\ldots=u_{n}=1$. Indeed, we then have

$$
\begin{aligned}
\phi_{p}(\alpha, u) & =\frac{1}{\sqrt[p]{2 n} 2^{n}} \sum_{\varepsilon \in \mathbb{D}_{n}}\left(\sum_{i=1}^{n}\left(1+\varepsilon_{i}\right)^{p}\right)^{1 / p} \\
& =\frac{1}{\sqrt[p]{2 n} 2^{n-1}} \sum_{k=0}^{n} \sqrt[p]{k}\left(\begin{array}{l}
n \\
k
\end{array}\right) .
\end{aligned}
$$


If the assumption in Proposition 4.1 were true for all $p>2$ with a fixed $n$, we could take the limit for $p \rightarrow 2$ to obtain

$$
\frac{1}{\sqrt{2 n} 2^{n-1}} \sum_{k=0}^{n} \sqrt{k}\left(\begin{array}{l}
n \\
k
\end{array}\right) \geqslant 1
$$

which is wrong for all $n \geqslant 1$.

Nevertheless, the next lemma shows, that if it is true for some $n$ and $p_{0}$, it is also true for all $p>p_{0}$ with the same $n$.

LEMMA 5.1. For fixed $u$ and $\alpha$ with $(9), \phi_{p}(\alpha, u) \in[0,1]^{n}$ is an increasing function of $p>2$, unless $u_{1}=\ldots=u_{n}=0$. In the latter case it is constant.

For the proof of this lemma we use the following more general result.

Proposition 5.2. Let $\phi_{1}, \ldots, \phi_{n}: I \rightarrow \mathbb{R}_{+}$be continuously differentiable functions on some interval $I \subset \mathbb{R}$ such that

$$
\phi_{i}(t) \log \phi_{i}(t) \leqslant t \phi_{i}^{\prime}(t) \quad \text { for } \quad t \in I \quad \text { and } \quad i=1, \ldots, n .
$$

Let $\alpha_{i}>0$ satisfy $\sum_{i=1}^{n} \alpha_{i}=1$. Then the function

$$
\phi(t)=\left(\sum_{i=1}^{n} \alpha_{i} \phi_{i}(t)\right)^{1 / t}
$$

is non-decreasing in $t \in I$. Moreover, if inequality (12) is strict for at least one $i=1, \ldots, n$ at each $t \in I$, then $\phi$ is increasing.

Proof: Since $\phi(t)>0$, the functions $\phi^{\prime}(t)$ and

$$
t^{2} \frac{\phi^{\prime}(t)}{\phi(t)}=t^{2} \frac{d}{d t}(\log \phi(t))
$$

are of the same sign. Hence $\phi(t)$ is non-decreasing provided that

$$
t^{2} \frac{d}{d t}(\log \phi(t))=t \frac{\sum_{i=1}^{n} \alpha_{i} \phi_{i}^{\prime}(t)}{\sum_{i=1}^{n} \alpha_{i} \phi_{i}(t)}-\log \sum_{i=1}^{n} \alpha_{i} \phi_{i}(t) \geqslant 0
$$

So we shall show that

$$
\left(\sum_{i=1}^{n} \alpha_{i} \phi_{i}(t)\right) \log \left(\sum_{i=1}^{n} \alpha_{i} \phi_{i}(t)\right) \leqslant t \sum_{i=1}^{n} \alpha_{i} \phi_{i}^{\prime}(t) \text { for } t \in I .
$$

Since the function $x \mapsto x \log x$ is convex on $\mathbb{R}_{+}$it is enough to verify that

$$
\sum_{i=1}^{n} \alpha_{i} \phi_{i}(t) \log \phi_{i}(t) \leqslant t \sum_{i=1}^{n} \alpha_{i} \phi_{i}^{\prime}(t) \text { for } t \in I
$$


But this follows immediately from (12). The moreover part is obvious from the preceding considerations.

Proof of Lemma 5.1: For fixed $\varepsilon \in \mathbb{D}_{n}$, we choose

$$
\phi_{i}(p)=\frac{\left(1+\varepsilon_{i} u_{i}\right)^{p}}{1+u_{i}^{p}} \text { for } i=1, \ldots, n .
$$

What we have to do is to show that condition (12) holds for this choice. Hence it is enough to prove the inequality

$$
\phi(p) \log \phi(p)<p \phi^{\prime}(p) \text { for } \quad p>0
$$

where $\phi(p)=(1+s)^{p} /\left(1+|s|^{p}\right)$ for some $s>-1, s \neq 0$. It is elementary to check that this inequality is equivalent to

$$
|s|^{p} \log |s|^{p}<\left(1+|s|^{p}\right) \log \left(1+|s|^{p}\right)
$$

which is true for $|s| \geqslant 1$ since the function $x \mapsto x \log x$ is increasing for $x \geqslant 1$ and which is true for $0<|s|<1$ since the left hand side is then negative whereas the right hand side is positive. Observe that in the case when $u_{1}=\ldots=u_{n}=1$, the functions $\phi_{i}(p)$ are constant only if $\varepsilon_{1}=\ldots=\varepsilon_{n}=-1$, so that the moreover part is also shown.

Finally, we want to get rid of $\alpha$. To this end, fix $u \in[0,1]^{n}, \alpha_{3}, \ldots, \alpha_{n}$ and consider varying $\alpha_{1}$ and $\alpha_{2}$. Since their sum $a=\alpha_{1}+\alpha_{2}$ is fixed by (9) we obtain that each of the summands

$$
\left(\sum_{i=1}^{n} \alpha_{i} \frac{\left(1+\varepsilon_{i} u_{i}\right)^{p}}{1+u_{i}^{p}}\right)^{1 / p}
$$

has the form $\sqrt[p]{\alpha_{1} s_{1}+\left(a-\alpha_{1}\right) s_{2}+t}$ for some $s_{1}, s_{2}, t \geqslant 0$. It can be easily checked that this is a concave function of $\alpha_{1}$, so it attains its minimum at one of the endpoints of the interval in which it can vary. So the same is true for $\phi_{p}(\alpha, u)$. Using this in an induction process yields that, for fixed $u \in[0,1]^{n}, \phi_{p}(\alpha, u)$ attains its minimum at some $\alpha$ for which all but one $\alpha_{i}=1 / 2 n$ and the remaining $\alpha_{i}=1 / 2+1 / 2 n$. Thus we have sharpened Theorem 4.1 as follows.

THEOREM 5.3. Let $q \geqslant p>2, n \in \mathbb{N}$ and $X=L_{q}$ or $X=l_{q}$. Let $\phi_{p}:[0,1]^{n} \rightarrow$ $\mathbb{R}_{+}$be defined by

$$
\phi_{p}(u)=\frac{1}{2^{n}} \sum_{\varepsilon \in \mathbf{D}_{n}}\left(\frac{1}{2 n} \sum_{i=1}^{n-1} \frac{\left(1+\varepsilon_{i} u_{i}\right)^{p}}{1+u_{i}^{p}}+\left(\frac{1}{2}+\frac{1}{2 n}\right) \frac{\left(1+\varepsilon_{n} u_{n}\right)^{p}}{1+u_{n}^{p}}\right)^{1 / p} .
$$

Assume that $\phi_{p}(u) \geqslant 1$ for all $u \in[0,1]^{n}$. Moreover, assume that $\phi_{p}(u)=1$ if and only if $u_{1}=\ldots=u_{n}=0$. Then

$$
\frac{1}{2^{n}} \sum_{\varepsilon \in \mathbf{D}_{n}}\left\|x_{\varepsilon}-y\right\| \geqslant \sqrt[q]{2}
$$


for all $x \in X$ with $n\|x\|^{q}=1$ and $y \in S(X)$, where the $x_{\varepsilon}$ are defined as in the beginning of this section (with $p$ replaced by $q$ ). Equality can occur only if the support of $x$ is (essentially) disjoint from the support of all the $y_{i}$ obtained from $y$ as above. In this case $X$ does not have the ADP.

\section{THE CASE $p=3$}

In this section, we show that the assumption of Theorem 5.3 holds for $n=2$ and $p=3$. This proves Theorem 1.1. What we have to show is the following lemma.

Lemma 6.1. The function

$$
\phi(u, v)=\frac{1}{4} \sum_{\varepsilon, \delta= \pm 1}\left(\frac{1}{4} \frac{(1+\varepsilon u)^{3}}{1+u^{3}}+\frac{3}{4} \frac{(1+\delta v)^{3}}{1+v^{3}}\right)^{1 / 3}
$$

satisfies the inequality $\phi(u, v) \geqslant 1$ for all $u, v \in[0,1]$. Moreover, equality holds only if $u=v=0$.

Despite of the fact that the graph of this function easily indicates the truth of the lemma, we were not able to give a 'nice' proof. Our proof works as follows. We first show by localisation that $\phi$ satisfies the claimed inequality in a sufficiently large neighbourhood of the point $u=v=0$. In the remaining region we rely for the checking of the inequality on interval arithmetics using the help of a computer. We only outline the proofs here in a way that they should be easily comprehensible by the reader. A computer algebra system would be of some help. All details of the computations are also to be found in [3]. The following two lemmas carry out these two steps and provide the full proof of Lemma 6.1.

Lemma 6.2. The above defined function $\phi$ satisfies $\phi(u, v) \geqslant 1$ for all $u, v \in$ $[0,1 / 10]$. Moreover, equality holds only if $u=v=0$.

Proof: It is easily checked, that the expressions

$$
\frac{(1+\varepsilon u)^{3}-\left(1+u^{3}\right)\left(1+3 \varepsilon u+3 u^{2}-2 u^{3}\right)}{u^{3}}
$$

are polynomials of degree 3 and 2 if $\varepsilon=1$ and $\varepsilon=-1$, respectively, which are positive for $u \in[0,1 / 10]$. Hence we have

$$
\frac{(1+\varepsilon u)^{3}}{1+u^{3}} \geqslant p(\varepsilon, u)=1+3 \varepsilon u+3 u^{2}-2 u^{3} \text { for } u \in[0,1 / 10] .
$$

Moreover, it can also be checked that, with

$$
q(t)=1+\frac{t}{3}-\frac{t^{2}}{9}+\frac{5 t^{3}}{81}-\frac{t^{4}}{16}
$$


the expression $\left((1+t)-q(t)^{3}\right) / t^{4}$ is a polynomial of degree 8 which is positive for $t \in[-2 / 5,2 / 5]$. This implies that

$$
\sqrt[3]{1+t} \geqslant q(t) \text { for } t \in[-2 / 5,2 / 5] .
$$

Using this together with $p(\varepsilon, u)-1 \in[-2 / 5,2 / 5]$ for $u \in[0,1 / 10]$, we obtain that

$$
\phi(u, v) \geqslant \frac{1}{4} \sum_{\varepsilon, \delta= \pm 1} q\left(\frac{p(\varepsilon, u)}{4}+\frac{3 p(\delta, u)}{4}\right) .
$$

The latter is a polynomial of total degree 12 in the two variables $u$ and $v$ of the form

$$
1+\frac{3 u^{2}}{16}+\frac{3 v^{2}}{16}-\frac{u^{3}}{6}-\frac{v^{3}}{2}+\sum_{4 \leqslant i+j \leqslant 12} \alpha_{i j} u^{i} v^{j}
$$

where 31 of the $\alpha_{i j}$ are negative. All these negative $\alpha_{i j}$ satisfy the inequality $-2 \alpha_{i j} \leqslant$ $10^{i+j-4}$ and can be divided into two groups of 16 and 15 members satisfying $i \geqslant 2$ and $j \geqslant 2$, respectively. We then conclude from $0 \leqslant u, v \leqslant 1 / 10$ that $\alpha_{i j} u^{i} v^{j} \geqslant-u^{2} / 200$ for the first group of $\alpha_{i j}$ and $\alpha_{i j} u^{i} v^{j} \geqslant-v^{2} / 200$ for the second group. Consequently, we arrive at

$$
\phi(u, v) \geqslant 1+\left(\frac{3}{16}-\frac{1}{60}-\frac{16}{200}\right) u^{2}+\left(\frac{3}{16}-\frac{1}{20}-\frac{15}{200}\right) v^{2} \geqslant 1+\frac{u^{2}+v^{2}}{10} .
$$

This finishes the proof of the lemma.

LEMma 6.3. The above defined function $\phi$ satisfies $\phi(u, v)>1$ for all $(u, v) \epsilon$ $[0,1]^{2} \backslash[0,1 / 10]^{2}$.

PROOF: We partition the area $(u, v) \in[0,1]^{2} \backslash[0,1 / 10]^{2}$ into rectangles and show for each rectangle $R=\left[u_{0}, u_{1}\right] \times\left[v_{0}, v_{1}\right]$ that $\phi(u, v)>0$ for $(u, v) \in R$. Since the function $(1+\varepsilon u)^{3} /\left(1+u^{3}\right)$ is increasing for $\varepsilon=1$ and decreasing for $\varepsilon=-1$ and $u \in[0,1]$, it is enough to show that $\psi\left(u_{0}, u_{1}, v_{0}, v_{1}\right)>0$ where $\psi$ is obtained from $\phi$ replacing the term $(1+\varepsilon u)^{3} /\left(1+u^{3}\right)$ with $\left(1+u_{0}\right)^{3} /\left(1+u_{0}^{3}\right)$ if $\varepsilon=1$ and with $\left(1-u_{1}\right)^{3} /\left(1+u_{1}^{3}\right)$ if $\varepsilon=-1$, and similarly for the terms involving $v$.

We used a partition into 4542 rectangles, which have to become smaller near the critical point $(0,0)$. We checked with Maple and with a C-program that the value of $\psi$ is bigger than $1 / 1000$ for each of these rectangles using a precision of at least 12 digits, thus eliminating roundoff errors. A Maple-worksheet containing the calculations is available from [3].

It should be clear from the preceding that it is possible, using $n=2$, to show that $l_{p}$ does not have the ADP for $p \geqslant p_{0}$ for some $p_{0}<3$. But, to come closer to $p=2$, it is necessary to increase the number of vectors involved, hence making the approach taken here computationally intractable. We also want to remark here that it is much easier to show Lemma 6.1 for $p=4$ instead of $p=3$. 


\section{THE RENDEZVOUS NUMBER OF COMPLEX $l_{\infty}$}

The purpose of this section is to prove Theorem 1.2. Observe that $S\left(l_{\infty}^{1}(\mathbb{C})\right)$ is isometric to $S\left(l_{2}(\mathbb{R})\right)$. Hence the rendezvous number of $l_{\infty}^{1}(\mathbb{C})$ is $4 / \pi$ as computed in [4].

Let us now assume that $d=2,3, \ldots, \infty$ and set $r=1 / 3+2 \sqrt{3} / \pi$. We first show that for any $x_{1}, \ldots, x_{n} \in S\left(l_{\infty}^{d}(\mathbb{C})\right)$ there exists $\phi \in[0,2 \pi]$ such that

$$
\frac{1}{n} \sum_{k=1}^{n}\left\|x_{k}-\exp (i \phi) e_{1}\right\| \leqslant r
$$

where $e_{1}$ is the first unit vector. To achieve this, it is clearly enough to prove the following lemma.

Lemma 7.1. For any $x \in \mathbb{S}\left(l_{\infty}^{d}(\mathbb{C})\right)$ with $d=2,3, \ldots, \infty$,

$$
\frac{1}{2 \pi} \int_{0}^{2 \pi}\left\|x-\exp (i \phi) e_{1}\right\| d \phi \leqslant r
$$

Proof: Let $\xi_{1}=r \exp (i \psi)$ be the first coordinate of $x$. Since

$$
\left\|x-\exp (i \phi) e_{1}\right\| \leqslant \max \left\{\left|\xi_{1}-\exp (i \phi)\right|, 1\right\}=\max \left\{\sqrt{1+r^{2}-2 r \cos (\phi-\psi)}, 1\right\},
$$

we obtain by rotational invariance that

$$
\frac{1}{2 \pi} \int_{0}^{2 \pi}\left\|x-\exp (i \phi) e_{1}\right\| d \phi \leqslant \frac{1}{2 \pi} \int_{0}^{2 \pi} \max \left\{\sqrt{1+r^{2}-2 r \cos \phi}, 1\right\} d \phi .
$$

Now we conclude from

$$
1+r^{2}-2 r \cos \phi \geqslant 1 \Longleftrightarrow 2 \cos \phi \leqslant r \Longleftrightarrow 1+r^{2}-2 r \cos \phi \leqslant 1+r-2 \cos \phi
$$

that

$$
\left\|x-\exp (i \phi) e_{1}\right\| \leqslant \frac{1}{2 \pi} \int_{0}^{2 \pi} \max \{\sqrt{2-2 \cos \phi}, 1\} d \phi=\frac{1}{\pi} \int_{0}^{\pi / 3} d \phi+\frac{1}{\pi} \int_{\pi / 3}^{\pi} \sqrt{2-2 \cos \phi} d \phi=r
$$

For $\phi, \psi \in[0,2 \pi]$, we let $x_{\phi, \psi}=\exp (i \phi) e_{1}+\exp (i \psi) \sum_{j=2}^{d} e_{j}$. Analogously as Lemma 7.1 shows that we can always arrange for an average distance at most $r$, the next lemma shows that we can also get an average distance at least $r$. Then Gross' Theorem tells us that $r$ is the unique rendezvous number of $S\left(l_{\infty}^{d}(\mathbb{C})\right.$ ) provided that $d$ is finite. Moreover, the intermediate value theorem shows that $r$ is also a rendezvous number of $S\left(l_{\infty}(\mathbb{C})\right.$ ). 
LEMMA 7.2. For any $x \in \mathbb{S}\left(l_{\infty}^{d}(\mathbb{C})\right)$ with $d=2,3, \ldots, \infty$,

$$
\frac{1}{4 \pi^{2}} \int_{0}^{2 \pi} \int_{0}^{2 \pi}\left\|x-x_{\phi, \psi}\right\| d \phi d \psi \geqslant r
$$

Proof: Let $x=\left(\xi_{k}\right)$. If $\left|\xi_{1}\right|=1$, we may assume by rotational invariance that $\xi_{1}=1$ and $\xi_{2}=r$ with $0 \leqslant r \leqslant 1$. Then

$$
\frac{1}{4 \pi^{2}} \int_{0}^{2 \pi} \int_{0}^{2 \pi}\left\|x-x_{\phi, \psi}\right\| d \phi d \psi \geqslant \frac{1}{4 \pi^{2}} \int_{0}^{2 \pi} \int_{0}^{2 \pi} \max \{|1-\exp (i \phi)|,|r-\exp (i \psi)|\} d \phi d \psi .
$$

If $\left|\xi_{1}\right|<1$, we fix $\varepsilon>0$ and choose $k \geqslant 2$ such that $1-\varepsilon<\left|\xi_{k}\right|=s \leqslant 1$. Again we may assume by rotational invariance that $\xi_{k}=s$ and $\xi_{1}=r$ with $0 \leqslant r \leqslant 1$. Then

$$
\begin{aligned}
\frac{1}{4 \pi^{2}} \int_{0}^{2 \pi} \int_{0}^{2 \pi}\left\|x-x_{\phi, \psi}\right\| d \phi d \psi & \geqslant \frac{1}{4 \pi^{2}} \int_{0}^{2 \pi} \int_{0}^{2 \pi} \max \{|r-\exp (i \phi)|,|s-\exp (i \psi)|\} d \phi d \psi \\
& \geqslant \frac{1}{4 \pi^{2}} \int_{0}^{2 \pi} \int_{0}^{2 \pi} \max \{|r-\exp (i \phi)|,|1-\exp (i \psi)|\} d \phi d \psi-\varepsilon
\end{aligned}
$$

Since $\varepsilon>0$ was arbitrary, we obtain in any case, by interchanging the roles of $\phi$ and $\psi$ in the latter one, that

$$
\begin{aligned}
\frac{1}{4 \pi^{2}} \int_{0}^{2 \pi} \int_{0}^{2 \pi}\left\|x-x_{\phi, \psi}\right\| d \phi d \psi & \geqslant \min _{0 \leqslant r \leqslant 1} \frac{1}{4 \pi^{2}} \int_{0}^{2 \pi} \int_{0}^{2 \pi} \max \{|1-\exp (i \phi)|,|r-\exp (i \psi)|\} d \phi d \psi \\
& =\min _{0 \leqslant r \leqslant 1} \frac{1}{\pi^{2}} \int_{0}^{\pi} \int_{0}^{\pi} \max \left\{2 \sin (\phi / 2), \sqrt{1+r^{2}-2 r \cos (\psi)}\right\} d \phi d \psi
\end{aligned}
$$

For $\psi \in[0, \pi]$ and $r \in[0,1]$, let us define $\alpha(r, \psi) \in[0, \pi / 2]$ by the equality $2 \sin (\alpha(r, \psi) / 2)=\sqrt{1+r^{2}-2 r \cos (\psi)}$. Then the inner integral in question evaluates to

$$
\begin{aligned}
\int_{0}^{\pi} \max \left\{2 \sin (\phi / 2), \sqrt{1+r^{2}-2 r \cos (\psi)}\right\} d \phi & =2 \int_{0}^{\pi} \max \{\sin (\phi / 2), \sin (\alpha(r, \psi) / 2)\} d \phi \\
& =4 f(\alpha(r, \psi) / 2)
\end{aligned}
$$

where

$$
f(\alpha)=\alpha \sin \alpha+\cos \alpha
$$


Then the assertion of the lemma follows if we can prove that, for $\psi \in[0, \pi / 2]$ and $r \in[0,1]$,

$$
F(r, \psi)=f(\alpha(r, \psi) / 2)+f(\alpha(r, \pi-\psi) / 2) \geqslant \pi / 6+\sqrt{3}
$$

Differentiating $F$ with respect to $\psi$ yields after a short computation that

$$
\frac{\partial F}{\partial \psi}(r, \psi)=r \sin \psi\left[\frac{\alpha(r, \psi)}{2 \sin (\alpha(r, \psi) / 2)}-\frac{\alpha(r, \pi-\psi)}{2 \sin (\alpha(r, \pi-\psi) / 2)}\right] .
$$

It can be easily checked that $\alpha(r, \psi) / 2 \sin (\alpha(r, \psi) / 2)$ is increasing in $\psi \in[0, \pi]$ if $r>0$. Hence the expression in brackets can vanish at most at one value of $\psi$, which happens at $\psi=\pi / 2$. So, for fixed $r>0, F(r, \psi)$ attains its minimum at $\psi=0$ or $\psi=\pi / 2$. Since $F(0, \psi)$ does not depend on $\psi$ the same is true for $r=0$. So we are left to check that $F(r, 0) \geqslant \pi / 6+\sqrt{3}$ and $F(r, \pi / 2) \geqslant \pi / 6+\sqrt{3}$ for all $r \in[0,1]$. In these cases we have

$$
F(r, 0)=\frac{1-r}{2} \arcsin \frac{1-r}{2}+\frac{1+r}{2} \arcsin \frac{1+r}{2}+\frac{\sqrt{3-r^{2}+2 r}}{2}+\frac{\sqrt{3-r^{2}-2 r}}{2}
$$

and

$$
F(r, \pi / 2)=2 f\left(\arcsin \sqrt{\frac{1+r^{2}}{4}}\right) .
$$

It takes another short computation to verify that both these functions are increasing in $r \in[0,1]$, so that they attain their minimum at $r=0$, which is indeed $\pi / 6+\sqrt{3}$ as wanted.

To finish the proof of Theorem 1.2 it remains to check that $r$ is the unique rendezvous number of $S\left(l_{\infty}(\mathbb{C})\right)$. To this end, define for some $N=1,2, \ldots$ vectors $x_{k}=\exp (2 \pi i k / N) e_{1}$ for $k=1, \ldots, N$. Let $x$ be a further vector in $S\left(l_{\infty}(\mathbb{C})\right)$. Since

$$
|\exp (i \phi)-\exp (2 \pi i k / N)| \leqslant 2 \pi / N \text { for } \phi \in[2 \pi(k-1) / N, 2 \pi k / N],
$$

we obtain from the triangle inequality that

$$
\begin{aligned}
\left\|x-x_{k}\right\| & \leqslant\left\|x-\frac{N}{2 \pi} \int_{2 \pi(k-1) / N}^{2 \pi k / N} e^{i \phi} d \phi e_{1}\right\|+\left\|\frac{N}{2 \pi} \int_{2 \pi(k-1) / N}^{2 \pi k / N} e^{i \phi} d \phi e_{1}-e^{2 \pi i k / N} e_{1}\right\| \\
& \leqslant \frac{N}{2 \pi} \int_{2 \pi(k-1) / N}^{2 \pi k / N}\left\|x-e^{i \phi} e_{1}\right\| d \phi+\frac{2 \pi}{N} .
\end{aligned}
$$

Averaging this over $k=1, \ldots, N$ and taking into account Lemma 7.1 yields

$$
\frac{1}{N} \sum_{k=1}^{N}\left\|x_{k}-x\right\| \leqslant r+\frac{2 \pi}{N} .
$$


Since $N$ was arbitrary, this shows that no rendezvous number of $S\left(l_{\infty}(\mathbb{C})\right)$ can be bigger than $r$. That no such number can be smaller than $r$ can be analogously verified using Lemma 7.2 instead of Lemma 7.1 and $N^{2}$ vectors

$$
x_{h, k}=\exp (2 \pi i h / N) e_{1}+\exp (2 \pi i k / N) \sum_{j=2}^{\infty} e_{j} \quad \text { for } \quad h, k=1, \ldots, N
$$

instead of the $N$ vectors $x_{k}$ above. So our proof of Theorem 1.2 is completed.

\section{REFERENCES}

[1] J. Cleary, A. Morris and D. Yost, 'Numerical geometry - numbers for shapes', Amer. Math. Monthly 93 (1986), 260-275.

[2] O. Gross, 'The rendezvous value of a metric space', Ann. of Math. Stud. 52 (1964), 49-53.

[3] A. Hinrichs, 'Details of some computations on the average distance property of $L_{p}$ ', http: //ww . minet . uni-jena.de/ hinrichs/adpcomp/.

[4] S.A. Morris and P. Nickolas, 'On the average distance property of compact connected metric spaces', Arch. Math. 40 (1983), 459-463.

[5] P.K. Lin, 'The average distance property of Banach spaces', Arch. Math. 68 (1997), 496-502.

[6] R. Wolf, 'On the average distance property of spheres in Banach spaces', Arch. Math. 62 (1994), 338-344.

Mathematisches Institut

FSU Jena

D 07743 Jena

Germany

e-mail: nah@rz.uni-jena.de
Current address:

Department of Mathematics

Texas A\&M University

College Station, TX 77843

United States of America 